\title{
Depressive Behavior of Rats Consuming Cocoa Powder and Cocoa Extract
}

\author{
Ariza Budi Tunjung Sari ${ }^{1)}$, Misnawi ${ }^{1)}$, Pratiwi Pudjiastuti ${ }^{2)}$, and Afaf Baktir ${ }^{2)}$ \\ ${ }^{1)}$ Indonesian Coffee and Cocoa Research Institute, J1. PB Sudirman 90, Jember 68118, Indonesia \\ ${ }^{2}$ Chemistry Department, Faculty of Science and Technology, Airlangga University, \\ Kampus C Mulyorejo, Surabaya 60115, Indonesia \\ ${ }^{*}$ Corresponding author: ariza.bts@iccri.net \\ Received: 17 October 2017 / Accepted: 17 April 2018
}

\begin{abstract}
Chocolate is associated with calm and happy feelings. However little is found to demonstrate if this effect is induced by active ingredient in cocoa rather than the sweetness of chocolate. In this study we tested the effect of cocoa on the depressive behavior of female rats suffering from estrogen deficiency. Twenty female rats aged seven weeks old were ovariectomized to remove the estrogen source. The rats were distributed into four groups and undergoing three day oral administration of $1 \mathrm{~g} / \mathrm{kg}$ body weight (bw) cocoa powder, $1 \mathrm{~g} / \mathrm{kg}$ bw cocoa extract, $1 \mathrm{mg} / \mathrm{kg}$ bw estradiol valerate and $1 \mathrm{~mL} / \mathrm{kg}$ bw vehicle (olive oil). Another group of rats having intact ovaries received $1 \mathrm{~mL} / \mathrm{kg}$ bw drinking water. The rats were undergoing forced swimming test (FST) one day before treatment and after treatment and the immobility time was recorded. No significant difference was found on the after-treatment immobility time across groups. However, all the rats in the vehicle group developed longer immobility time after-treatment compared with the result from before-treatment test, suggesting that depression had occurred in ovariectomized rats receiving no active substances. In the other hand, very few rats had greater after-treatment immobility time in the cocoa powder and estradiol valerate groups. In the cocoa extract group, all the rats had shorter immobility time after treatment when it is compared with before treatment time. We conclude that compounds in cocoa have certain extent of estrogenic activity that affects emotional state.
\end{abstract}

Keywords: Cocoa, estrogen, depression, forced swimming test, immobility

\section{INTRODUCTION}

A well-known attribute of chocolate is to trigger positive mood states i.e. happy and calm (Meier et al., 2017). This is however being unclear whether this attribute comes from a compound in cocoa, or the comforting sweetness and smooth texture of chocolate itself (Hormes, 2014). A study by Rozin et al. (1991) reported the chocolate consumption pattern among female chocolate consumers. It was found that the intention on eating chocolate was increasing when they were entering menstruation phase. Considering the low estrogen level around menstruation time, we propose a relationship between cocoa and estrogen, thus chocolate eating can compensate the discomforts arise during estrogen depletion. 
In our previous study, we investigated the estrogenic activity of cocoa powder and extract through anuterotrophic assay, in which the test articles were applied in estrogen-deficient rats for three days and the weight of uterus was observed (Sari et al., 2017). In the same trial we conducted a forced swimming test before and after cocoa administration. Forced swimming test is a procedure to detect depressive behavior in rodent. The animal is placed in water for certain time frame in which a normal rat will move vigorously to survive. A situation when the rat does a minimal movement only to keep the head above the water is considered immobility, which is indicating depressive behavior (Castagné et al., 2011).

In this study, we evaluate immobility time of ovariectomized rats consuming cocoa powder, cocoa extract, and olive oil as vehicle and compared them with rats receiving semisynthetic estradiol and intact ones. A test article is considered carrying anti-depressant potential if it is able to reduce immobility time. It is expected to reveal the anti-depressant potential in the cocoa product and propose the underlying mechanism.

\section{MATERIALS AND METHODS}

The cocoa powder was prepared from commercial dried cocoa beans. After roasting, the shell was removed and fat was expressed out of the bean. The remaining cocoa solids were then ground and sieved to produce cocoa powder. The cocoa extract was obtained from fresh cocoa beans through ethanol maceration. The liquid was concentrated and dried to yield cocoa extract powder. Positive control used estradiol valerate (Progynova, Bayer). All the test substances were dissolved in olive oil, and accordingly olive oil was used as negative control.
Twenty five female wistar rats, aged five weeks at arrival, were allocated in collective cages (3-4 individuals per cage) and acclimatized for two weeks. The ovariectomy procedure was performed when the rats had reached seven weeks old. Prior to the surgery, rats were injected with $0.1 \mathrm{~mL}$ ketamine and $0.1 \mathrm{~mL}$ xylazine for anesthesia. The fur was clipped and the skin was incised. The procedure removed both ovaries. Five rats underwent sham operation, in which the similar surgery was conducted without any tissue removed so that the ovaries were kept intact. Administration of test articles was started two weeks after surgery. The treatment has been approved by ethical committee of Faculty of Veterinary, Airlangga University (certificate no. 620-KE).

The test articles comprised of cocoa powder (CP) $1 \mathrm{~g} / \mathrm{kg}$ bw, cocoa extract (CE) $1 \mathrm{~g} / \mathrm{kg}$ bw, estradiol valerate (EV) as the positive control $1 \mathrm{mg} / \mathrm{kg}$ bw, and olive oil (OO) as negative control $1 \mathrm{~mL} / \mathrm{kg}$ bw. The sham operated rats $(\mathrm{SH})$ received drinking water $1 \mathrm{~mL} / \mathrm{kg}$ bw. The test articles were treated to the rats via oral route once a day for three consecutive days.

Forced swimming tests (FST) were conducted one day before treatment and on the third day after the last treatment. Water $\left(29-30^{\circ} \mathrm{C}\right)$ was prepared in a transparent chamber made from high density polyethylene plastic ware. The depth of water was approximately $25 \mathrm{~cm}$, longer than rat body length. The rats followed training session 24 hours before the first test, in which the rats swam for 15 minutes. On the testing session, the swimming lasted for 5 minutes and the movement was constantly taped using video camera. Immobility is defined as a situation when rat makes minimal movement only to keep the head above the water, rather than actively swimming, diving or climbing. The total time in the 5 minute swimming session 
that was spent in immobility was recorded as immobility time in seconds.

The immobility time from five individuals per group were then calculated for mean, standard deviation, normality (Saphirowilk's) and heterogenity (Levene's) test. Analysis of variance was performed using $95 \%$ level of confidence, $\alpha=0.05$. The effect of test article is considered significant when $P$ value is smaller than $\alpha$.

\section{RESULTS AND DISCUSSION}

The immobility time of each test group is displayed in Table 1. The before-treatment immobility time was calculated before test articles applied, while the after-treatment time was taken from the test conducted in the day 3. The time difference is the beforetreatment time subtracted with the aftertreatment time. The negative time difference indicates reduction in the immobility time after treatment, compared with that of beforetreatment. Animal with greater after-treatment time, thus the time difference is positive, showed a longer time spent in an immobile condition and was considered depressive.

The immobility time across groups before treatment was not significantly different. Some studies found that depressive symptom in mammals, did not occur immediately after ovariectomy. It typically takes around 4 weeks until immobility time showed apparent increase (Li et al., 2014). This may explain the insignificant difference between olive oil and sham operated groups. Similarly, the immobility time in the post treatment test also was not significant across groups, which is demonstrated also in the time difference. Some studies applied four week course of treatment to reach a detectable effect, thus the three day treatment may had not resulted noticeable outcomes.

Despite the test articles failed to bring significant changes in the immobility time, the treatments had produced different number of depressive rats between groups. All rats in the olive oil group had greater immobility time after-treatment, while only one rat in the cocoa powder group and in the estradiol valerate group showed increasing immobility time after-treatment. No rat in the cocoa extraxct group had an increased immobility time after being applied with cocoa extract. This indicates the potential of cocoa powder and cocoa extract to deliver anti-depressive activity, eventhough a longer duration of treatment is required to produce a clear result.

Forced swimming test is a widely used method to evaluate the depressive behavior in rodent. Rat response towards water emulates human reaction towards stress, which involves struggling, learning, and despairing. At the first time a rat gets in contact with water, it will struggle and move vigorously by climbing and swimming. After several minutes, it will eventually learn that doing nothing will keep its head afloat. The learning process is done

Table 1. Immobility time (s) of rats in each test group, calculated before, and after administration of test articles

\begin{tabular}{lcccc}
\hline \multirow{2}{*}{ Test articles } & \multicolumn{3}{c}{ Immobility time $(\mathrm{s})^{*}$} & $\begin{array}{c}\text { Number of } \\
\text { depressiverats in group }\end{array}$ \\
\cline { 2 - 4 } Cocoa extract & Before treatment & After treatment & Time difference & 0 \\
Cococ powder & $207.60 \pm 43.4$ & $149.40 \pm 88.72$ & $-58.20 \pm 50.91$ & 1 \\
Estradiol valerate & $218.60 \pm 26.3$ & $191.00 \pm 57.61$ & $-27.60 \pm 40.00$ & 1 \\
Olive oil & $227.80 \pm 28.3$ & $183.60 \pm 47.59$ & $-44.20 \pm 69.27$ & 5 \\
Sham operated & $208.00 \pm 32.6$ & $223.40 \pm 21.55$ & $15.40 \pm 12.32$ & 2 \\
\hline
\end{tabular}

Notes: *mean \pm standard deviation 
$24 \mathrm{~h}$ before the test. In the testing session, despaired rat will immediately show less movement upon placement in water (Castagné et al., 2011).

The forced swimming test has been incorporated in the investigation of depression drug of choices such as fluoroxetine and paroxetine (Detke et al., 1995), and also novel glutamatergic drugs (Haj-Mirzaian et al., 2014). Estradiol valerate also exerts anti-depressive activity (Romano-Torres \& Fernandez-Guasti, 2010) and had ever been patented as antidepressant (Itil et al., 1982). It decreases immobility time in female ovariectomized mice during forced swimming test through inhibition of nitric oxide (Heydarpour et al., 2013).

Estrogen deficiency is one factor triggering depression. It occurs on women approaching menopause, as well as those undergone ovariectomy(Georgakis et al., 2016). The mechanism of estrogen towards mood is by modulating nerve cell generation and signaling. Estrogen has two types of receptors i.e. alpha and beta types, and alpha receptor is responsible for controlling depression (Murakami et al., 2015). The effect of cocoa compound toward estrogen or alpha receptor is less studied, but there is hypothesis that cocoa polyphenol promotes synthesis of alpha receptor protein (Oleaga et al., 2012). The ability of cocoa extract to decrease the number of depressive rats in estrogen deficiency model warrants further research on the relationship between cocoa active compounds and estrogen receptors.

\section{CONCLUSION}

Neither cocoa powder nor cocoa extract had successfully decreased the mean immobility time in ovariectomized rats. However, those two substances had reduced the number of depressive animals. The proposed mechanism is involving estrogenic pathway.

\section{REFERENCES}

Castagné, V.; P. Moser; S. Roux \& R.D. Porsolt (2011). Rodent models of depression: Forced swim and tail suspension behavioral despair tests in rats and mice. Current Protocols in Pharmacology, $55,11-18.10$.

Detke, M.J.; M. Rickels \& I. Lucki (1995). Active behaviors in the rat forced swimming test differentially produced by serotonergic and noradrenergic antidepressants. Psychopharmacology, 121, 66-72.

Georgakis, M.K.; T.P. Thomopoulos; A.-A. Diamantaras; E.I. Kalogirou; A. Skalkidou; S.S. Daskalopoulou \& E.T. Petridou (2016). Association of age at menopause and duration of reproductive period with depression after menopause: a systematic review and metaanalysis. JAMA Psychiatry, 73, 139-149.

Haj-Mirzaian, A.; S. Ostadhadi; N. Kordjazy; A.R. Dehpour \& S.E. Mehr (2014). Opioid/NMDA receptors blockade reverses the depressant-like behavior of foot shock stress in the mouse forced swimming test. European Journal of Pharmacology, 735, 26-31.

Heydarpour, P.; M. Salehi-Sadaghiani; M. JavadiPaydar; R. Rahimian; G. Fakhfouri; M. Khosravi; S. Khoshkish; M.H. Gharedaghi; M. Ghasemi \& A.R. Dehpour (2013). Estradiol reduces depressive-like behavior through inhibiting nitric oxide/cyclic GMP pathway in ovariectomized mice. Hormones and behavior, 63, 361-369.

Hormes, J. (2014). Perimenstrual chocolate craving: from pharmacology and physiology to cognition and culture. pp. 61-70. In: Handbook of Diet and Nutrition in the Menstrual Cycle, Periconception and Fertility. Wageningen Academic Publishers, The Netherland.

Itil, T.M.; G. Laudahn \& W.M. Herrmann (1982). Use of estradiol valerate as an anti-deppresant. Patent No. US4330538 A. Schering Aktingesselschoft, Berlin, Germany. 
Li, L.H.; Z.C. Wang; J. Yu \& Y.Q .Zhang (2014). Ovariectomy results in variable changes in nociception, mood and depression in adult female rats. PloS one, 9, 94312.

Meier, B.P.; S.W. Noll \& O.J. Molokwu (2017). The sweet life: The effect of mindful chocolate consumption on mood. Appetite, 108, 21-27.

Murakami, G.; Y. Hojo; M. Ogiue-Ikeda; H. Mukai; P. Chambon; K. Nakajima; Y. Ooishi; T. Kimoto \& S. Kawato (2015). Estrogen receptor $\mathrm{KO}$ mice study on rapid modulation of spines and long-term depression in the hippocampus. Brain research, 1621, 133-146.

Oleaga, C.; M. García; A. Solé; M. IzquierdoPulido \& V. Noé (2012). CYP1A1 is overexpressed upon incubation of breast cancer cells with a polyphenolic cocoa extract. European Journal of Nutrition, 51, 465-476.
Romano-Torres, M. \& A. Fernandez-Guasti (2010). Estradiol valerate elicits antidepressant-like effects in middle-aged female rats under chronic mild stress. Behavioural pharmacology, 21, 104-111.

Rozin, P.; E. Levine \& C. Stoess (1991). Chocolate craving and liking. Appetite, 17, 199-212.

Sari, A.B.T.; Misnawi; P. Pudjiastuti \& A. Baktir (2017). Uterus weight of ovariectomized rats given cocoa powder and extract. Pelita Perkebunan, 33, 45-50. 